\title{
LAS RELACIONES ENTRE GENERACIONES EN UNIVERSIDADES: LA PERSPECTIVA DE LOS PROFESORES
}

\author{
Diego Barragán ${ }^{1}$ e Reinaldo dos Santos iD2
}

\section{Resumo}

O artigo analisa as relações entre as gerações que participam dos processos educativos nas universidades a partir da perspectiva dos professores. As condições predominantes nas universidades, os possíveis elos que podem ser tecidos, as ações alternativas que podem ser realizadas, estão moldando as experiências e as visões dos professores sobre suas relações com os estudantes. Foi utilizada metodologia qualitativa, foi mantido um diário de campo e foram realizadas entrevistas em profundidade em universidades públicas e privadas em Bogotá. O balança de poder estabelecido nas instituições influencia as relações, especificamente, entre estudantes e professores. Foram encontrados três tipos: autoritário, enquadrado na tradição onde o balança de poder é orientado para o professor que recebe apoio institucional, com diretrizes de treinamento estabelecidas e distante dos alunos. As conflituosas são orientadas para manter um número significativo de estudantes; o balança de poder é inclinado para os estudantes, estabelecendo uma relação relativamente forte com os proprietários ou administradores da universidade; onde os processos de formação e os professores ocupam lugares marginais. E os formativos mostram um equilíbrio no balança de poder, onde professores e alunos desenvolvem suas funções tendo como núcleo o processo educacional e a consolidação de uma comunidade acadêmica; a participação de grupos fora do ensino ou da pesquisa não é importante. Três alternativas são apresentadas onde as relações entre gerações são influenciadas pela dinâmica e lógica dos grupos de poder encontrados nas universidades.

Palavras-chave: Gerações; Relações; Professores; Alunos; Universidades.

\section{AS RELAÇÕES INTERGERACIONAIS NAS UNIVERSIDADES: A PERSPECTIVA DOS PROFESSORES}

\section{Resumo}

O artigo analisa as relações entre as gerações que participam dos processos educativos nas universidades a partir da perspectiva dos professores. As condições predominantes nas universidades, os possíveis elos que podem ser tecidos, as ações alternativas que podem ser realizadas, estão moldando as experiências e as visões dos professores sobre suas relações com os estudantes.

\footnotetext{
${ }^{1}$ Pós-Doutorado em Educação, Universidade Federal da Grande Dourados, Brasil. Doutorado em Ciências Sociais, Universidad de Buenos Aires, Argentina. Professor Universitário e Pesquisador Independente em Bogotá, Colombia.

${ }^{2}$ Pós-Doutorado em Educação, Universidade de São Paulo, Brasil. Doutorado em Sociologia, Universidade Estadual Paulista Júlio de Mesquita Filho. Professor Associado e Pesquisador da Universidade Federal da Grande Dourados, Brasil.
} 
Foi utilizada metodologia qualitativa, foi mantido um diário de campo e foram realizadas entrevistas em profundidade em universidades públicas e privadas em Bogotá. O balança de poder estabelecido nas instituições influencia as relações, especificamente, entre estudantes e professores. Foram encontrados três tipos: autoritário, enquadrado na tradição onde o balança de poder é orientado para o professor que recebe apoio institucional, com diretrizes de treinamento estabelecidas e distante dos alunos. As conflituosas são orientadas para manter um número significativo de estudantes; o balança de poder é inclinado para os estudantes, estabelecendo uma relação relativamente forte com os proprietários ou administradores da universidade; onde os processos de formação e os professores ocupam lugares marginais. E os formativos mostram um equilíbrio no balança de poder, onde professores e alunos desenvolvem suas funções tendo como núcleo o processo educacional e a consolidação de uma comunidade acadêmica; a participação de grupos fora do ensino ou da pesquisa não é importante. Três alternativas são apresentadas onde as relações entre gerações são influenciadas pela dinâmica e lógica dos grupos de poder encontrados nas universidades.

Palavras-chave: Gerações; Relações; Professores; Alunos; Universidades.

\section{INTERGENERATIONAL RELATIONS IN UNIVERSITIES: THE PERSPECTIVE OF THE PROFESSORS}

\section{Abstract}

The article analyses the relationships between the generations involved in educational processes in universities from the perspectives of the professors. The conditions prevailing in universities, the possible links that can be forged, the alternative actions that can be taken, shape the experiences and visions of teachers about their relations with students. Qualitative methodology was used, a field diary was kept and in-depth interviews were conducted in public and private universities in Bogotá. The balance of power established in institutions influences relations specifically between students and professors. Three types were found: the authoritarian ones, framed in the tradition where the balance of power is oriented towards the professor who receives institutional backing, with established training guidelines and distant from the students. The conflictual ones are oriented towards maintaining a significant number of students, the balance of power is tilted towards the students establishing a relatively strong relationship with the owners or administrators of the university; where training processes and professors occupy marginal places. And the formative ones show a equilibrium in the balance of power, where professors and students develop their functions with the educational process and the consolidation of an academic community at the core; the participation of groups outside teaching or research is not important. Three alternatives are presented in which the relations between generations are influenced by the dynamics and logics of the power groups found in universities.

Keywords: Generations; Relationships; Professors; Students; Universities. 


\section{Introdução}

Las relaciones de las personas que intervienen en procesos educativos están condicionadas por los contextos institucionales y sociales donde se desarrollan; específicamente, se manifiestan en las funciones que cumplen profesores y estudiantes que participan en universidades (ELIAS, 1982, p.29). Las relaciones de quienes participan en procesos educativos pueden estar condicionadas por múltiples factores, acá se destaca el encuentro entre generaciones (ELIAS, 1998; SARAT, 2014, p.97). Cada vez, las distancias entre grupos crecen, los referentes simbólicos, culturales y sociales establecen abismos en las relaciones entre jóvenes y adultos. Incluso, las relaciones pueden complicarse cuando se guían por manuales o convenciones que tienen las personas sobre el desempeño adecuado de los estudiantes y las pautas aceptadas para sus relaciones con los profesores; enmarcándose en unas condiciones reglamentarias que, en ocasiones, se encuentran lejos de las dinámicas que pueden tener las relaciones, independiente de entenderlas o no, las reglamentan, dan los marcos para su realización (ELIAS, 1998, p.418).

Las relaciones entre profesores y estudiantes se basan en encuentros entre grupos que pertenecen a mundos distintos, que deben desarrollar un proceso de formación, durante algunos años, que parte de sus interacciones, sus relaciones, sus construcciones, sus acuerdos, sus desacuerdos y sus hábitos (KAPLAN, 2016, p.116). En estas relaciones, intervienen grupos que hacen parte del quehacer cotidiano en la universidad donde se evidencia una balanza de poder (ELIAS, 2011, p. 26): directivos, funcionarios públicos o privados, miembros de grupos económicos, religiosos o políticos (HUNGER, ROSSI \& SOUZA, 2011). En el caso de los profesores, las interacciones con estos grupos les permiten desarrollar experiencias adecuadas a los medios donde habitan, actúan y desarrollan sus funciones de docencia e investigación (SANTOS, 2009, p.157). Estas relaciones no son homogéneas, implican conflictos, las pautas de conducta que se desarrollaran son distintas en correspondencia al espacio. Al acercarse un poco a las realidades del presente, se necesita indagar por herramientas que permitan dar cuenta de los problemas actuales tomando el proceso social donde participan y el acumulado de conocimientos disponible (ELIAS, 2009, p.338).

En los escenarios universitarios se presentan conflictos, uno evidente, es el encuentro entre generaciones (ELIAS, 1998; SARAT, 2009); se crea una brecha entre jóvenes que entran a la universidad representando el presente y profesores que, hipotéticamente, representan el pasado (BLANCO \& PIERELLA, 2009, p.73). Ambos grupos se encuentran en espacios sociales, temporales, simbólicos y económicos, es un encuentro entre "grupos difícil de sortear, [...] experiencia en la que cada vez comienza a ocupar un lugar residual la transmisión del pasado para dar paso a la habitación de un presente del que los jóvenes se sentían dueños y la construcción de un futuro en el que lo viejo no tendría cabida" (PIERELLA, 2009, p.8). No es sólo el encuentro con los estudiantes, los encuentros con las personas o grupos cercanos a la administración que representan poder y dinero en algunas universidades $y$, 
especialmente, su participación significa influencia en el entorno social donde los profesores habitan (BARRAGÁN, 2018a).

Las personas se ven inmersas en situaciones, momentos, contextos, encuentros, condiciones, problemas y relaciones que desbordan su capacidad de control (ELIAS, 1990; GOFFMAN, 1991). El profesor no puede controlar totalmente el desarrollo de sus actividades o tener siempre relaciones fuertes con quienes interactúan. Ahora, en las relaciones indirectas, como las virtuales, el control sobre los procesos de formación se dificulta, pero el profesor debe generar un vínculo para realizar su actividad y el cumplimiento de sus tareas. Se genera una relación entre las personas creando una interdependencia funcional entre las personas y los procesos donde participan (ELIAS, 1990, p.68). Los profesores hacen parte de un proceso educativo que comparten con otros grupos, donde en el desarrollo se van construyendo las lógicas, las interacciones y las dinámicas con las personas que participan, las instituciones o los entornos sociales ofrecen espacios para la realización de estas relaciones (ELIAS, 1998, p.435).

En algunas instituciones, generalmente privadas, las relaciones entre estudiantes y profesores no son el núcleo del proceso educativo, lo importante son los encuentros de los estudiantes con los representantes de los grupos de poder de la universidad (empresarios, políticos o religiosos); los profesores pueden ocupar lugares marginales (BARRAGÁN, 2018a). Se presentan grupos de poder que utilizan la universidad, incluso escuelas y colegios, para reproducir sus ethos de comunidad, sus pautas de actuación, sus rituales, sus prácticas, sus cargas de valores; que pueden estar cerca o lejos del proceso educativo (BARRAGÁN, 2018). Estos grupos, con intereses privados, proponen una alternativa para garantizar su reproducción a través de la educación universitaria, la consolidación en el entorno social y en el largo plazo de su comunidad, proyectando su permanencia o posicionamiento en la cotidianidad o en espacios que consideran estratégicos (BOURDIEU \& PASSERON, 2009).

En la actualidad, los jóvenes se transforman en su paso por la universidad, entran siendo adolescentes de 15 a 18 años y salen como adultos jóvenes de 18 a 21. Evidencian cambios en las relaciones con casi todos los grupos sociales con los que tienen contacto, no sólo con padres o profesores; ahora, pueden participar de redes sociales presenciales o virtuales que influyen en sus relaciones (DE GRANDE, 2013). Y desde luego, las condiciones y los entornos sociales donde se encuentran, donde viven, los envuelven. Lo complejo es que los adultos no los entienden, el camino más transitado, es establecer unas condiciones que cumplir y el joven se evalúa en la cercanía o el alejamiento de las condiciones. Los adultos no entienden, lo que hacen es acercarlos al modelo establecido de joven que deben cumplir (ELIAS, 1998, p.438), lejos de las personas que asisten a las aulas en el proceso educativo.

En sus experiencias cotidianas, los profesores van desarrollando encuentros con personas o grupos que tienen relación con sus actividades en la universidad y necesitan compartir con ellos para cumplir propósitos concretos (GOFFMAN, 2006). Existen otros encuentros que son indirectos, puede ser virtuales, donde la presencia física no cuenta, pero que sirven para realizar sus 
actividades en la universidad donde pueden utilizar medios tecnológicos que ayudan a la comunicación y a los lazos que tejen las personas. En especial, cuando participan de un proceso educativo que implica una duración, un tiempo, unas actividades, unas evaluaciones, unos compromisos y unos resultados; los encuentros, directos 0 indirectos, se presentan como los contactos que desarrollan las personas durante su experiencia profesoral (BARRAGÁN \& SANTOS, 2020; CARRILLO, 2015).

Pertenecer a grupos distintos, incluso un poco extraños, puede generar barreras, problemas y conflictos (ELIAS, 1998, p.416). En algunos lugares, estos grupos tienen esquemas cognitivos, pero son precarios, no tienen unas bases intelectuales que permitan contrastar con marcos explicativos o referenciales para entender su situación, deben esperar que los otros personajes interpreten, expliquen y, en ocasiones, que decidan por ellos (WACQUANT, 2010). Así, las personas pueden estar expuestas a situaciones donde se orientan según las pautas establecidas por individuos cercanos o por los medios a los que se encuentran vinculados. Entonces, las personas están inmersas en situaciones inmediatas que no pueden controlar, no cuentan con herramientas cognitivas para entenderlas y enfrentarlas (ELIAS, 1990, p.19); lo que hacen las personas es desarrollar estrategias que les permita desenvolverse (GOFFMAN, 1991), en las situaciones donde se encuentran, tratan de optar por, lo que ellos consideran, el mejor camino.

Incluso, participar en redes cercanas a la docencia, puede exponer a las personas a formas de ver, observar, pensar y actuar, que pueden adquirir o no, en correspondencia a su decisión o a las condiciones donde estén inmersas (HUNGER, ROSSI \& SOUZA, 2011); "...es decir, las redes tienen dinámicas que la persona observa y toma la decisión de vincularse o asumirlas en la medida que evalúesu utilidad, su pertenencia para el desempeño de su labor, el criterio parte de su experiencia" (BARRAGÁN, 2018a, p.62). Adicional, se puede señalar la participación en grupos de diversidad política, académica, sexual, cultural (BLANCO \& PIERILLA, 2009); es decir, es la participación en redes de intercambios la que les posibilita a los profesores estar en contacto con las personas que fortalecen su ejercicio profesional (BARRAGÁN, 2018).

El profesor construye su experiencia en docencia e investigación a partir de su trayectoria y debe desarrollar sus relaciones aprendiendo a desenvolverse según las pautas que se establecen institucional y socialmente; son procesos que pueden durar varios años. Si realiza algo, una acción que no se acepte en quienes intervienen, que no sea apta para los profesores, puede tener problemas; incluso, puede ser expulsado del proceso (ELIAS, 1998). Cada grupo puede tener sus condiciones, o múltiples opciones de relacionarse. Aquí, se observa cómo se desarrollan los tópicos evidentes que señalan dentro de las relaciones un sólo grupo, los profesores, llevadas a cabo en diferentes universidades, facultades, comunidades y condiciones objetivas. Se intentan señalar las clases de relaciones entre estudiantes y profesores en universidades públicas y privadas de Bogotá. 


\section{Metodologia}

El artículo se realizó siguiendo un enfoque cualitativo (ROCKWELL, 2015), acercándose a los protagonistas de los procesos educativos (BJØRNDAL, 2020). Una dificultad en el levantamiento de la información fue el "encuentro entre iguales", entrevistar, hablar, compartir y observar a profesores; que pertenecían a diferentes campos académicos y laboraban en distintas universidades, pero manifestaban sus inquietudes sobre sus relaciones con estudiantes, su desempeño frente a los procesos de formación, su lugar en la universidad y las herramientas teóricas 0 metodológicas consideradas pertinentes para comprenderlas (BARRAGÁN \& SANTOS, 2020). Los diálogos y los testimonios fueron interesantes, para sistematizarlos se utilizaron dos herramientas que evidenciaban las experiencias de los participantes (GOFFMAN, 2006, p.15): entrevistas en profundidad a los profesores y un diario de campo donde se registró la observación participante realizada en los espacios donde se desarrolló la investigación (BECKER, 2009).

El trabajo de campo se realizó durante tres meses (noviembre, diciembre de 2019 y enero 2020) participaron 16 profesores, 8 mujeres y 8 hombres, 6 magíster y 10 doctores; de la Facultad de Ciencias Sociales-FCS, Facultad de Ciencias Económicas-FCE y Facultad de Ciencias Básicas e Ingeniería - CBeI, de Universidades Públicas-UP y Universidades Privadas-UPV de Bogotá. Para procesar la información se utilizó el software Atlas.ti versión 8. Siguiendo a Carli (2012, p.95), se eligieron "personas del común"; profesores que pueden encontrarse cotidianamente en las universidades desempeñando su labor ${ }^{3}$; que tuvieran,al menos, contratos temporales que implicaba 40 horas a la semana, fueron 11 , y 5 con contratos indefinidos.Destacando, las vinculaciones temporales para los profesores universitarios, que participan en procesos de formación de mediana y de larga duración, es frecuente en países de América Latina (RIKAP, 2016; LOPEZ; ARIADNA; GARCÍA; et al, 2016).

\section{Resultados e discussões}

El acercamiento a la relación entre profesores y estudiantes partió de las interdependencias constantes entre las formulaciones teóricas y hallazgos empíricos (ELIAS, 2011). El desarrollo empírico partió de las entrevistas y la observación participante, teniendo como referente la ciudad, las dinámicas de las universidades que se estudiaron, los grupos de poder que influían y, en especial, las experiencias de los profesores. Después del procesamiento de la información, se encontraron tres categorías que ayudan a sistematizar las experiencias y los testimonios de los profesores, son herramientas para organizar el desarrollo argumentativo, pero las categorías no son excluyentes entre sí, una persona puede tener cabida en dos o tres tipos de relaciones. Las categorías que se utilizaron para explicar las relaciones entre profesores y estudiantes fueron: las autoritarias, las conflictivas y las formativas. 
Las autoritarias son relaciones caracterizadas por la tradición, donde las dinámicas institucionales depositan un grado significativo de poder en los docentes, generando un desequilibrio en la relación, sustentado en: la trayectoria docente, los logros profesionales o académicos, y en los vínculos o participación en grupos de poder que guían la universidad. Los estudiantes juegan un rol pasivo, presentándose como depósitos de conocimientos, obligados a cumplir con las exigencias académicas, deben aprender comportamientos adecuados y a desenvolverse de acuerdo con las lógicas de las relaciones que reproduce el docente e imperan en la universidad.

Son recurrentes los señalamientos de los entrevistados de las brechas que imperan en las relaciones entre estudiantes y profesores, y cuál es la vía recurrente, utilizada por algunos profesores, para tratar esta distancia. Violeta (FCE-UP) sostiene:

[...] hay una brecha... sé cómo se expresan los profesores de ellos. Hemos tenido reuniones donde los profesores se expresan muy feo de los estudiantes... los estudiantes no tienen criterio para evaluarme, los estudiantes todo lo quieren fácil, los estudiantes ya no quieren estudiar, los estudiantes son unos vagos... las niñas son unas mentirosas e insinuadoras, por eso dicen que las acosan... ese es el imaginario sobre esa brecha generacional.

Carlos, (FCS-UP), continua en esta vía:

[...] siento que cada vez más los estudiantes tienen menos ideas y contexto, o sea no conocen su historia, no conocen la biografía, no saben escribir, no saben analizar... digamos como que llegan cada vez con menos elementos críticos, con menos elementos para leer la realidad y para tomar decisiones, como que son cada vez más unos tecnócratas, como que cada vez la educación está formándolos para ser más dóciles, para ser más obedientes y menos críticos...

Es un alejamiento de los estudiantes de los espacios y los procesos básicos que deben tener para desarrollar la formación. Siguiendo con las intervenciones, se estableció una representación de los estudiantes donde no tienen un compromiso con su desempeño, con sus estudios, con su rol en la universidad. En algunos casos, no cumplen con las condiciones mínimas para su permanencia en la institución, en ocasiones esto no importa. El esfuerzo, la constancia, la dedicación de parte de los estudiantes no se evidencia en sus labores académicas y menos en sus relaciones con los profesores.

Duarte (FCE-UP), profundiza en la diferencia entre generaciones, sostiene que cada día el problema toma mayor fuerza:

[...] los profesores corresponden a diferentes generaciones, tal vez los mayores chocan mucho con los estudiantes... a punto de pensionarse, de 55 (años) para arriba, son quienes se educaron con un modelo muy tradicional, bajo unos esquemas de 
relacionamiento...tradicional... ahí si yo veo un abismo... Digamos con profesores más jóvenes se cierra un poquito la brecha, pero sigue existiendo...

Para una profesora o un profesor de una edad superior a los 55 años, las relaciones con jóvenes les genera una enorme dificultad; incluso, pueden pertenecer a mundo diferentes. Laura (FCE-UPR), desarrolla un argumento en el mismo sentido, los profesores de:

[...] 60 años en adelante dictándole a muchachos que pueden tener 17 años y ahí la desconexión es importante porque los profesores no han comprendido que estas generaciones tienen otras dinámicas de aprendizaje o formas de relacionamiento grupal. Entonces, empieza a convertirse casi que en un trauma tanto para los muchachos que no pueden convivir con el método de enseñanza del profesor y para los profesores porque digamos que ...tienen todavía una lectura de pura obediencia respeto a los estudiantes y la lógica de los jóvenes no son de la obediencia a la que estaban acostumbrados los abuelos... creo, hay una desconexión medio perjudicial...

El encuentro de estas generaciones en el aula de clase, en un proceso educativo, genera un conflicto importante que recae en las relaciones autoritarias. Fuera del encuentro entre mundos distintos, los grupos participan en un proceso de aprendizaje, rutinas e interacciones que pueden durar, un semestre o varios años. Al no encontrar unas condiciones adecuadas, el proceso de aprendizaje y las relaciones se convierten para los participantes en un suplicio, un constante y permanente sufrimiento, donde sólo quieren que termine pronto. No importa la vinculación con el conocimiento, su cercanía a las comunidades académicas o profesionales, su vinculación con la universidad; aquí, lo importante es cerrar estos episodios que se convierten en pesadillas para los profesores y los estudiantes. El proceso de formación y la relación no importan, lo importante es terminar.

Adicional, los elementos básicos como leer, escribir, operaciones aritméticas o cumplir con las obligaciones, fueron mencionados, constantemente, como fuentes de tensión en las relaciones entre las generaciones; especialmente, los conflictos que generan las relaciones autoritarias donde los profesores se presentan como depositarios del saber, del poder y de la autoridad. No tener las competencias mínimas dificulta la relación y, como práctica extendida, identificada por varios participantes de la investigación, el plagio se convierte en usual. Roberto (FCS-UPV), vincula un elemento importante en la relación entre generaciones respecto al proceso de formación, cómo la tecnológica afecta negativamente el desempeño de los estudiantes:

[...] yo creo que hay una dificultad grande y es la tecnología, que en vez de favorecer... ha disminuido la calidad de la educación, se usa mal. La internet influye mucho en el nivel educativo de los estudiantes, un estudiante se puede distraer estudiando siempre, 
no importa que tenga, pero si tenemos la distracción a la mano en dos segundos se va a distraer mucho más... la posibilidad de plagiar se amplía, cada vez es más difícil detectar el plagio... no los pongo a escribir porque voy a revisar y es plagio, el estudiante también se acostumbró a eso: inclusive, sin pensar que está mal, inclusive ya es su forma de estudiar, es tomar un texto parafraseado y eso se dificulta un poco.

La cantidad de información disponible en internet ha generado que los estudiantes utilicen el plagio como una herramienta usual; soportado un poco por el número de estudiantes que deben atender los profesores ${ }^{4}$.

Las siguientes son las relaciones conflictivas. Igual que en las anteriores, se presentan desequilibrios donde los grupos de poder que dominan o administran las universidades depositan la mayor participación y una mayor cuota de poder en los estudiantes; en especial, en instituciones que necesitan mantener un número importante de estudiantes para garantizar recursos económicos o para promover la vinculación a comunidades políticas, religiosas o económicas 5 . Se establece una relación directa entre los propietarios o los administradores de las instituciones con los estudiantes, que serían sus consumidores y donde los profesores ocupan funciones de acompañamiento o lugares secundarios. Esto genera un conflicto fuerte de los profesores, sus labores y sus contactos con los dueños de las universidades en el desarrollo de los procesos de formación y el establecimiento de sus relaciones con los estudiantes. Se ataca, se desvalora la figura y las funciones del profesor. No importa el proceso educativo que desarrolla, lo importante es cómo se relaciona con los grupos de poder.

Aquí, se hace la reproducción de un modelo donde los profesores son fichas intercambiables en un tablero, el proceso académico queda a la deriva. Estos grupos, rompen la relación básica entre profesores y estudiantes, para presentar a los estudiantes como miembro de una comunidad, donde adelantan un proceso de formación, ven asignaturas con algunos profesores; pero, los importantes son quienes representan a los grupos de poder que son dueños 0 administradores de la universidad. La relación entre estudiantes y profesores toma otras vías. Violeta (FCE-UP), da una ilustración,

[...] la universidad les ha vendido que ellos son el cliente, esa relación de cliente y proveedor de servicios y tengo que satisfacer al cliente, ellos ya están empoderados en ese mensaje... la misma universidad les ha dicho. He estado en escenarios donde en la inducción, quehace la institución, les dice ustedes son los clientes y nosotros vamos a hacer todo para satisfacer sus necesidades...la relación con el profesor es muy compleja porque cuando te enfrentas a esos escenarios, donde el estudiante te dice es que yo soy el cliente y usted tiene que satisfacer esas necesidades, pues es manejar esta relación, pues porque esta es la única relación en 
la que usted paga y usted no es el cliente, es un individuo de la sociedad en el cual la universidad le tiene que responder es cierto... se han presentado situaciones... no solamente en... los de pregrado, sino con mucha más fuerza a los de posgrado... a los de pregrado todavía les puedes plantear la reflexión de que están en un proceso... Pero el de posgrado, yo creo que entra con el imaginario, sin generalizar por supuesto, que el pagar le van a dar el cartón al final, que no importa si estoy haciendo el proceso de formación de la manera adecuada... sino que con pagar, asistir a clase es igual a cartón (diploma)...

Angela (PCS-UPV), menciona situaciones que son recurrentes de su labor docente a nivel de postgrado:

[...] temporalmente es que cada vez hacen maestría más chiquitos...creo que eso no les da el criterio suficiente para saber que quieren hacer en la vida, son muy consentidos... pretenciosos, ahorita ellos quieren un cinco fácil... Yo soy muy exigente... el estudiante también cambia de una universidad a la otra, los estratos diferentes de una población de estudiante que tengan mayores ingresos, mayores posibilidades económicas, cambiar de públicas a privada... en esa medida el perfil del estudiante es muy diferente, históricamente muy pequeños, muy consentidos, de verdad, se ofenden muy fácil y la formación resulta muy difícil. Aparte de eso, está el negocio, de lo que es la matrícula y la competencia a nivel profesional... a veces ni siquiera podemos hacer selección y todos pueden entrar y en esa medida el estudiante ve que todo va a ser muy fácil y no se pueden quedar, porque si el estudiante pierde... van a pelear, eso sí es algo que me indigna de la academia. No sé si me quedemucho tiempo, porque de verdad eso no me gusta, ese problema, anteriormente por ejemplo, los estudiantes de la (otra universidad privada), yo si los sentía incluso hace dos años mucho más respetuosos a los de acá y tengo un amigo que trabaja en (otra universidad privada) y me dice que es peor allá, respecto a (esta universidad) cada vez más dinero, son más pretenciosos y tú eres el empleado.

Laura (PCE-UPV), destaca el papel asignado a los profesores dentro de las relaciones conflictivas:

[...] deja de verse al maestro como maestro y se empieza a ver como un empleado que se le paga por un servicio, el cliente siempre tiene la razón, el estudiante es el cliente... eso ha calado el asunto de mercantilizar la educación porque se le refuerza la idea al estudiante de que él está pagando por un servicio y el profesor no tiene una función distinta a suministrarle lo que necesita, además a pasarlo, eso ha degenerado el proceso de formación desde hace una década.

Los lugares marginales de los profesores en las relaciones conflictivas hacen que respondan con las necesidades de las personas que orientan las 
universidades o los requerimientos de los jóvenes, y no como la figura garante de un proceso de formación, sino como un empleado que acompaña el desempeño de aprendices dentro de una institución.

Justo (FCS-UPV), suma otro elemento a la relación conflictiva, que cada vez tienen mayor presencia en la formación en la universidad: la desmotivación. Presente en el proceso, en la institución y lo que los jóvenes observan en internet o en su realidad:

[...] exactamente, las problemáticas actuales que se ven en su generación, entonces yo intento hacer eso, para cerrar un poco esa brecha... yo tengo un hijo de esa edad, entiende uno ese lenguaje y entiende la supuesta desmotivación, porque es una generación que yo la veo un poco desmotivada, ¿Cómo es?, quiero ser youtuber, ¿para qué una carrera de 5 años?, pues ya los que están por lo menos tienen un interés, pasan el filtro y quieren estar en la universidad, que es el problema que se está viendo con la caída del número de estudiantes, las universidades ya no, es un bajonazo... no mucha paciencia, no están comprometidos para hacer una carrera de 5 años.

La inquietud es asumir un proceso de formación de 5 o más años en la universidad con lo que implica en esfuerzo, en recursos, en relaciones y en tiempo; las preguntas de algunos jóvenes ¿para qué asumir un proceso de este tipo? Todos los días, a cada momento, les presentan opciones de formación que se encuentran lejanas a las universidades, por los medios masivos de comunicación; opciones que son de corto tiempo, con competencias específicas $y$, algunas, con validez internacional. Independiente de su valor o no, repetirlo por todos los medios posibles, a cada momento, hacer publicidad de estos negocios, hacen que las personas pongan en la balanza su participación en la universidad.

Derivada de lo anterior, Oriana (FCE-UPV) señala una diferencia en la forma, en la actitud con que la mayoría de los jóvenes asumen los procesos académicos:

Uno no va a poder regañar un chico como lo hacía hace 20 años, uno podía sancionar a un chico, nada que ver la forma de sanción es diferente... yo le puedo decir a alguien mire usted cometió plagio, tiene 0 y pierde la materia... Ahora dicen: bueno perfecto, me tengo que ir de la universidad pues me voy... ya uno no tiene muchas formas de control, antes había controles mínimos... ahora...el mayor control es la expulsión y a veces a la gente no le interesa.

Las formas de regulación, de sanción para la validación de los procesos académicos han cambiado, se han modificado de acuerdo con las expectativas de los jóvenes, si cometen un error, no cumplen con una actividad, simplemente: no importa. Incluso, frente a la pérdida de condición de estudiantes, otra universidad puede recibirlo y terminar su formación 
universitaria o cansarse y retirarse, no importa. María (FCS-UPV), sostiene que en la actitud, la respuesta de los jóvenes frente a los procesos de formación y las posibilidades de vida, existe un elemento ideológico que ayuda a comprenderlo:

[...] se ha generado cierta distancia con los chicos... yo creo, tengo una inquietud, una sospecha de que...hubo un elemento ideológico intencional para que los jóvenes no logren organizar teoría y práctica, una cosa es lo que les dicen y otra cosas es lo que se hace... siento que es una intencionalidad de lo educativo, de lo religioso y como de otros sectores, porque no puede ser posible nosotros tenemos...120 estudiantes semestrales, de esos 120 por ahí 20 logran relacionar y articular mejor...uno dice algo tuvo que haber pasado en el proceso de vida de estos jóvenes que llegan a la universidad y no logran hacer las articulaciones...

La situación de estos jóvenes hace pensar, según María, de un alejamiento de su realidad, de la poca o nula necesidad de entender qué les pasa y qué sucede en el entorno social donde se encuentran. Simplemente, están lejos, no entienden y no importa.

Además, no tienen los elementos básicos para cumplir con las condiciones mínimas durante su proceso de formación. Roberto (FCS- UPV), señala:

[...] el proceso universitario hoy... uno sigue llevando esa carga de la mala lectura, de la mala escritura, porque las universidades no dan las herramientas para que el estudiante pueda superar esas condiciones y lo que le van a generar es un problema y nos va a generar problema como sociedad... porque, al final la gente se va a graduar y eso no es de esta universidad, es de todas las universidades. Se van a graduar con esas dificultades...

A pesar de las limitaciones, de los problemas de formación básicos de los estudiantes se van a graduar, van a terminar la universidad. Esto sostiene que los estudiantes puedan aprobar las asignaturas con esfuerzos mínimos. Entonces, las relaciones conflictivas se afincan en la vinculación de la universidad al mercado, representado en lemas como: "el cliente siempre tiene la razón"; los estudiantes no tienen las herramientas básicas para desarrollar su formación y asumen el proceso con desmotivación. El hecho importante es que los jóvenes paguen y hagan parte de una comunidad religiosa, política o económica; la labor docente queda al margen. No importan el proceso de formación, los encuentros y las relaciones entre profesores y estudiantes van a estar atravesadas por los conflictos potenciados por los dueños o dirigentes de las universidades.

Por último, las relaciones formativas son la base del encuentro entre las generaciones (ELIAS, 1998, p.450). En el proceso educativo los profesores y los estudiantes desempeñan funciones básicas, cada uno tiene su rol, interdependiente de los otros, para desarrollar su proceso, destacando la aproximación a tradiciones de conocimiento, a comunidades académicas que instauran dinámicas de comportamiento para sus miembros. Estas tres 
funciones pueden incluir otras, son las bases para la relación entre profesores y estudiantes. Las relaciones son equilibradas en el sentido que cada grupo desempeña sus funciones, lo mejor posible, como parte del proceso educativo que involucra a todos. Desde luego, las condiciones institucionales ayudan al desarrollo de la relación, pero el epicentro es el encuentro profesores y estudiantes.

Estas relaciones son difíciles, en algunos contextos institucionales se han generado las condiciones para el desarrollo de la relación formativa; pero, lo interesante es cómo vincular a personas que pertenecen a mundos distintos a un proceso educativo y que participen activamente en su desarrollo. Carlos (FCS-UP), sostiene:

[...] yo he tenido que transformarme como docente... para mí eso ahora es fundamental, digamos que tiene que ser un espacio vital donde la gente, donde yo veo seres humanos y no cifras...el estudiante ideal no existe... mientras que si yo voy dispuesto a descubrir quién está ahí... yo he aprendido de los estudiantes cantidad de cosas...y de todas las cosas que me ha tocado inventarme han sido por los estudiantes... yo trato de entenderlos a ellos para entender porque ellos tienen las dificultades que tienen o las facilidades que tienen... cómo le digo, no se trata de ser como ellos, sino de reconocer que yo tengo una diferencia y que entre nosotros podemos aportarnos.

La dificultad radica en poder establecer las condiciones adecuadas, establecer las dinámicas de la relación donde profesores y estudiantes participen, según Carlos (FCS-UP), Helga (FCBeI-UP) y la mayoría de profesores participantes, es el escollo importante que se debe sortear. Cada profesor en correspondencia a su área de actuación, su trayectoria y sus estrategias trata de establecer una relación significativa con los estudiantes. Helga (FCBeI-UP), señala un espacio institucional donde los estudiantes los evalúan, en especial el aporte que han recibido para su formación y para su vida:

[...] siempre se invita a los estudiantes a que hagan esa evaluación....Una pregunta muy bonita dentro de la evaluación de los estudiantes... preguntan sí el haber cursado la asignatura conmigo les ha hecho cambios en su vida, y todas las veces han contestado que sí, que han tenido cambios significativos en su vida; en términos del análisis, en términos de la ética, en términos de las implicaciones que tienen para su formación personal haber tomado esa asignatura...

En el proceso se crea una relación formativa, donde los estudiantes tienen inquietudes frente a lo que aprenden, a lo que hacen, a sus actuaciones y las manifiestan a los profesores que son quienes deben trazar el camino adecuado para que estas personas avancen en su formación. No sólo cumpliendo los requisitos mínimos, se avanza descubriendo mundos distintos en los cuales ellos pueden hacer parte y el profesor debe proponerles caminos. Lucio (FCBeI-UPV), 
plantea que, usualmente, se establece una barrera entre profesor y estudiantes, que gradualmente, el proceso de aprendizaje puede destruir creando relaciones entre los participantes como representantes de un campo de saber y de una comunidad,

[...] otra cosa interesante me he dado cuenta con mi carrera docente y es que cuando está el personaje, profesor o docente y el personaje estudiante, siempre hay como una barrera inherente ... Pero a medida que tú vas relacionándote con ellos y los vas tratando como personas que están aprendiendo y que tú también puedes aprender de ellos, rompes esa barrera...los chicos ya no te temen, y tú también no te crees acá arriba, hay un equilibrio... cuando se genera esa relación hay confianza y respeto mutuo...Ellos se sienten más en confianza para preguntar las cosas y eso te hace sentir mejor, porque sientes que lo estás haciendo bien.

Lucio (FCBeI-UPV), resalta la formación como el núcleo de las relaciones entre profesores y estudiantes, es el vínculo entre generaciones que pertenecen a mundos distintos. Son relaciones que se construyen, trayendo diversos tipos de elementos, desde simples a complejos; donde lo importante es el proceso educativo y la participación de los actores centrales tratando de hacer su desempeño lo mejor posible. Las relaciones no son fáciles.

El encuentro lo que hace es tratar de ligar personas que pertenecen a mundos distintos. La relación se basa en una constante ida y vuelta (ELIAS, 1990), entre la exigencia que se hace entre los participantes. Roberto (FCSUPV), sostiene,

[...] hay estudiantes que uno ve un cambio impresionante de primero a último semestre, y sigue siendo la posibilidad de movilidad social... Pero uno debe contribuir para que se siga dando, a mí me gusta mucho estar en el aula, hay reflexiones que se dan desde lo cotidiano, que es su primera mirada del mundo...es muy rico ir discutiendo lo cotidiano en las clases, debatiéndolo, como se ve el mundo, desde una profesión, desde una mirada política o teórica... Creo que en eso la mayoría es receptivo, ayudan los tiempos, el contexto, seguramente en su casa ya se empezaron a cuestionar, por el paro, por la crisis, empiezan escuchar y dicen: uy este me lo está explicando teóricamente; lo que estamos viendo en la calle... siento que sigue habiendo mucha receptividad.

Las idas y vueltas, implica vincular los conocimientos que están descubriendo los estudiantes reflexionando sobre la cotidianidad donde se encuentran. Es tratar de presentar y apropiar elementos que contribuyan al entendimiento de hechos, de acciones, de personas o de problemas que envuelven a los estudiantes y que pueden tener, desde el encuentro con el profesor, las herramientas para entender lo que sucede en su entorno. La receptividad relaciona los elementos que se presentan en el saber como las 
situaciones donde se encuentran los jóvenes, potencian las herramientas que disponen para pensar y actuar en su vida cotidiana.

María (FCS-UPV), propone desde los profesores elementos para soportar las relaciones formativas:

[...] yo siempre quería ser maestra y para mí ser maestra es como el encuentro con el otro, la posibilidad de romper cosas. Por ejemplo, cuando los chicos llegan y porque tú ves eso así es como entender esa lógica y yo les planteo como otras lógicas y los chicos dicen y yo no había visto eso.... Es esa posibilidad yo no había visto eso profesora y veo el mundo... esto me ayuda a no ser racista, a no ser clasista, a entender, a no darme pena ser pobre, porque a muchos les da pena ser pobres.

Las relaciones formativas se sostienen en la posibilidad de desarrollar un proceso educativo, vinculando dos generaciones, donde una propone lecturas desde sus ámbitos académicos para comprender lo que acontece en su cotidianidad, y la otra trata de tomar los elementos, que considera valiosos, para su formación, para entender su cotidianidad. Dos generaciones que intentan compartir lecturas alternativas del mundo a partir de sus interdependencias.

\section{Considerações finais}

La alternativa de investigación utilizada en el artículo fue acercarse un poco a las realidades del presente, a las personas que participan, indagar con herramientas que permitan dar cuenta de los problemas, tomando el proceso social donde están inmersos y el acumulado de conocimientos disponible (ELIAS, 2009). Las relaciones entre profesores y estudiantes se encuentran influenciadas por las dinámicas imperantes en los contextos sociales $y$, especialmente, institucionales donde se desarrollan (ELIAS, 1982; SARAT, 2009; BARRAGÁN \& SANTOS, 2020). Hipotéticamente, aquí se habla de personas interdependientes que participan en lugares distintos dentro de procesos educativos (HUNGER, ROSSI, \& SOUZA, 2011) que tienen la posibilidad de un acercamiento al conocimiento, a actitudes frente a la cotidianidad, la participación en comunidades especializadas y la adopción de pautas de comportamiento en correspondencia con estos requerimientos. La relación básica se establece entre profesor y estudiantes; pero, su intensidad varía en correspondencia con las dinámicas institucionales y los grupos de poder que dominan las universidades; en especial, en donde hacen presencia grupos económicos, religiosos y políticos (HONORATO, 2017). Las presencias e intermediaciones de estos grupos establecen diferencias significativas en las relaciones que se tejen, que se manifiestan en universidades públicas y universidades privadas; desde luego, las relaciones no pueden ser homogéneas, las pautas de conducta que se desarrollaran son distintas.

En correspondencia a las condiciones que se observaron en las universidades, se evidenció que se presenta la balanza de poder en el desarrollo de las relaciones entre las generaciones que intervienen en el proceso educativo 
(ELIAS, 1998). Las lógicas y dinámicas en cada contexto institucional, las relaciones que se forman entre estudiantes y profesores (BARRAGÁN, 2018), la participación de los grupos de poder, generalmente en universidades privadas, en las funciones y las lógicas que se desarrollaban, se pudieron identificar de tres tipos de relaciones: las autoritarias, las conflictivas y las formativas.

Las autoritarias, basadas en la formación tradicional donde la balanza de poder tiene un desequilibrio otorgando el protagonismo a los profesores, quienes se proponían como los depositarios del conocimiento, de las condiciones adecuadas, son quienes actúan en correspondencia de un particular tipo de profesores y que con su participación activa en comunidades académicas o profesionales, son representantes de los grupos de poder que dominan la institución. Manteniendo una barrera entre grupos. Los estudiantes tienen papeles secundarios, simplemente son recipientes que se deben llenar, deben adoptar comportamientos en correspondencia con las dinámicas institucionales y, acatando los lineamientos preestablecidos por los grupos de poder, pueden terminar su proceso educativo. En ocasiones, sin vincularlo significativamente a su formación, a sus actuaciones, a su vida; sólo terminan siguiendo las pautas establecidas y ya.

Las conflictivas, evidencian un desequilibrio donde el poder de la relación se encuentra en los estudiantes y los representantes, administrativos o dueños, de los grupos de poder, principalmente en universidades privadas. Sólo cuando los profesores son representantes o hacen parte de los grupos de poder tienen un reconocimiento de su papel, o de lo contrario, los profesores que estén lejos del poder ocupan lugares marginales en los procesos educativos, en la vida y en las dinámicas institucionales. Además, por su papel marginal pueden ser reemplazados en un semestre, un periodo o un año, son fichas intercambiables dentro de un tablero (RIKAP, 2016). Los vínculos fuertes que generan los estudiantes no son con la comunidad académica representada en los profesores, sus vínculos se generan con los miembros de las comunidades económicas, políticas o religiosas que dominan la universidad.

Las últimas, son las formativas donde se generan equilibrios en las interacciones funcionales entre profesores y estudiantes, potenciando los procesos de formación a partir de que cada grupo asume y desempeña su labor. Es una posibilidad para el encuentro de generaciones que pertenecen a mundos distintos, donde se presentan alternativas de comprensión a su realidad con herramientas distintas y donde los estudiantes hacen parte de comunidades que ofrecen pautas alternativas de comportamiento y de actuación. Ubicar su lugar en la relación hace que las comunidades académicas se construyan como parte de un proceso educativo, donde cada grupo tiene un lugar y desempeña su función dentro de las interacciones. Donde el pertenecer a una comunidad, realizar ciertas prácticas, acercarse a los conocimientos acumulados, a rituales significativos; los hace parte de una comunidad que desarrolla procesos académicos y el encuentro entre generaciones lo potencia.

Se debe aclarar, las relaciones entre profesores y estudiantes, entre generaciones (SARAT, 2009), tienen papeles importantes, especialmente en contextos institucionales 0 sociales donde se presentan conflictos y 
desigualdades (PIERELLA, 2009). Las personas están inmersas en situaciones que no pueden controlar, no cuentan con herramientas cognitivas para entenderlas y enfrentarlas (ELIAS, 1990); lo que hacen es desarrollar estrategias que les permita desenvolverse (GOFFMAN, 1991), ante las situaciones donde se encuentran, tratan de optar por el mejor camino, por la ruta trazada eficazmente en la universidad. Los profesores hacen parte de este esfuerzo en el proceso educativo, van construyendo las lógicas, las interacciones y las dinámicas con las personas que participan en espacios donde pueden desarrollarlas (ELIAS, 1998).

Por último, es necesario indagar un poco sobre las transformaciones de las relaciones entre profesores y estudiantes en contextos institucionales y sociales distintos. Las dificultades que están teniendo las relaciones entre jóvenes y adultos, hasta el punto de tener distancias y estar obligados a compartir una comunidad, establecen la necesidad de continuar con la indagación, en especial, el desarrollo de investigaciones. Como parte del proceso en las relaciones entre estudiantes y profesores se pueden identificar rasgos estructurales, pero con los cambios actuales es necesario continuar profundizando sobre ¿cómo desarrollar relaciones equilibradas en grupos que pertenecer a tiempos y a mundos distintos?

\section{REFERÊNCIAS}

BARRAGÁN, DIEGO. La experiencia estudiantil en una sociedad hostil. Una aproximación a los estudiantes universitarios de Ibagué (2012). Bogotá: Universidad Externado de Colombia, 2018.

BARRAGÁN, DIEGO. Un acercamiento a los profesores universitarios: experiencia, figuraciones y escenarios. Intermeio: Revista Do Programa De Pós-Graduação Em Educação -Universidade Federal de Mato Grosso do SulUFMS. 24 (48), pp. 55-70, 2018a.

BARRAGÁN, DIEGO \& DOS SANTOS, REINALDO. Los profesores universitarios desde el legado de Norbert Elias. Una revisión de literatura. En: Norbert Elias em debate: usos e possibilidades de pesquisa no Brasil.[livro eletrônico]. Ana Flavia Braun Vieira; Miguel Archanjo de Freitas Junior (Orgs.). Ponta Grossa: Texto e Contexto, 2020. (Coleção Singularis, v.6). pp 564-584.

Disponible en:

https://www.textoecontextoeditora.com.br/produto/detalhe/norbert-elias-emdebate-usos-e-possibilidades-de-pesquisa-no-brasil/42

BECKER, HOWARD. Trucos del oficio: cómo conducir su investigación en ciencias sociales. Buenos Aires: Siglo XXI, 2009

BJØRNDAL, CATO. Student teachers 'responses to critical mentor feedback: A study of face-saving strategies in teaching placements. Teaching and

Teacher Education, 91, p. 103047, 2020. 
BLANCO, RAFAEL. \& PIERILLA, MARÍA. Experiencias estudiantiles en la universidad contemporánea. Notas acerca de modos de abordaje de los discursos sobre autoridad, sexualidad y afectividad. Educación, lenguaje y sociedad. $6(6) ; 69-84,2009$.

BOURDIEU, PIERRE. \& PASSERON, JEAN. Los herederos. Los estudiantes y la cultura. Buenos Aires: Siglo XXI, 2009.

CARLI, SANDRA. El estudiante universitario. Hacia una historia del presente de la educación pública. Buenos Aires: Siglo XXI, 2012.

CARRILLO, CARMEN. Retos del profesorado universitario: experiencias profesorales de una docente e investigadores novel en el ámbito de la formación del profesorado. Profesorado: revista de curriculum y formación de profesorado. 19 (2): 303-317, 2015.

DE GRANDE, PABLO. Aportes de Norbert Elias, Erving Goffman y Pierre Bourdieu al estudio de las redes personales. Andamios. 10 (22); 237-258, 2013.

ELIAS, NORBERT. Scientific Establishments. In: Elias, Norbert; Martins, Herminio, \& Whitley, Richard. Scientific Establishments and Hierarchies. D. Reidel Publishing Company, Dordrecht, Holland. pp.3-70, 1982.

ELIAS, NORBERT. Compromiso y distanciamiento. Ensayos de sociología del conocimiento. Barcelona: Ediciones Península, 1990.

ELIAS, NORBERT. La civilización de los padres. Ed: Wailer, V: La civilización de los padres y otros ensayos. Bogotá: Editorial Norma. p. 407-450, 1998.

ELIAS, NORBERT. Los Alemanes. Buenos Aires: Nueva Trilce, 2009.

ELIAS, NORBERT. Sociología Fundamental. Barcelona: Gedisa, 2011.

GOFFMAN, ERVING. Los momentos y sus hombres. Barcelona: Paidos, 1991.

GOFFMAN, ERVING. Frame Analysis. Los marcos de la experiencia. Madrid: CIS-Siglo XIX, 2006.

HONORATO, TONY. A Reforma Sampaio Dória: Professores, Poder e Figurações. Educação \& Realidade, vol. 42, p.1279-1302, 2017.

HUNGER, DAGMAR., ROSSI, FERNANDA \& SOUZA, SAMUEL. A teoria de Norbert Elias: uma analise do ser professor. Educação e Pesquisa. 37 (4); 697-710, 2011. 
KAPLAN, CARINA. El racismo de la violencia. Aportes desde la sociología figuracional. En C. KAPLAN y M. SARAT (Comp.) Educación y procesos de civilización. Miradas desde la obra de Norbert Elias. Buenos Aires: Facultad de Filosofía y Letras, Universidad de Buenos Aires, pp. 99-118, 2016.

LÓPEZ, ARIADNA. GARCÍA, OMAR. PÉREZ, RICARDO. MONTERO, VIRGINIA. ROJAS, ELSA. "Los profesores de tiempo parcial en las universidades públicas estatales: una profesionalización inconclusa". Revista de la Educación Superior. 45 (180), 23-39, 2016.

PIERELLA, MARÍA. Acerca de la autoridad de los profesores universitarios. Una aproximación a los discursos de estudiantes de la Universidad Nacional de Rosario en el tiempo presente. Ponencia. Universidad de Buenos Aires, Instituto Gino Germani, 2009.

ROCKWELL, ELSIE. La experiencia etnográfica. Historia y cultura en los procesos educativos. Buenos Aires; Paidos, 2015.

RIKAP, CECILIA. "Heterogeneities and working conditions among academics at the University of Buenos Aires: A comparative study between the Pharmacy and Biochemistry, and the Economics' Schools". Trabajo y Sociedad. 27, 109-137, 2016.

SANTOS DOS, REINALDO. Família e escola no processo contemporâneo de socialização primária: Reflexão sociológica sobre representações e expectativas institucionais. En: Tempos e espaços civilizadores: diálogos com Norbert Elias. DARI \& SARAT (Orgs.). Dourados, MS: Editora da UFGD. pp. 155-176, 2009.

SARAT, MAGDA. Relações entre gerações e processos "civilizadores". En: Tempos e espaços civilizadores: diálogos com Norbert Elias. DARI \& SARAT (Orgs.). Dourados, MS: Editora da UFGD. pp. 105-120, 2009.

SARAT, MAGDA. Educación de la infancia: acercamientos entre Brasil y Argentina. Historia de la Educación, Anuario SAHE. 15 (2), 93-118, 2014.

WACQUANT, LOÏC. Parias urbanos: Marginalidad en la ciudad a comienzos del milenio. Buenos Aires: Manatial, 2010.

Recebido em: 05 de março de 2021.

Aceito em: 31 de março de 2021. Publicado em: 30 de junho de 2021. 